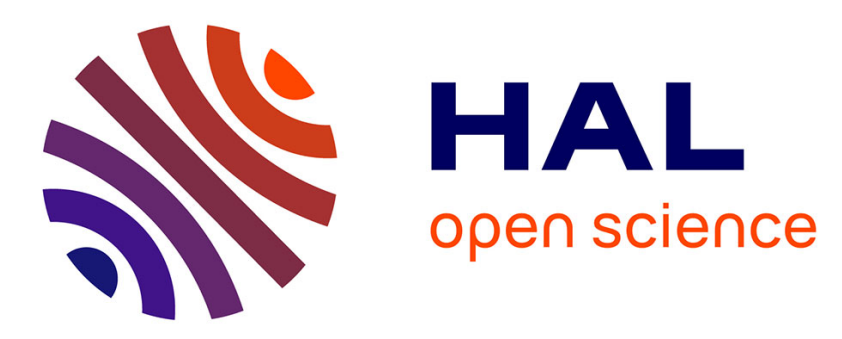

\title{
Performance of a digital transmitter leakage LMS-based cancellation algorithm for multi-standard radio-frequency transceivers
}

Robin Gerzaguet, Laurent Ros, Fabrice Belveze, Jean-Marc Brossier

\section{- To cite this version:}

Robin Gerzaguet, Laurent Ros, Fabrice Belveze, Jean-Marc Brossier. Performance of a digital transmitter leakage LMS-based cancellation algorithm for multi-standard radio-frequency transceivers. Digital Signal Processing, 2016, Elsevier Digital signal Processing, 51, p. 35-46. 10.1016/j.dsp.2016.01.009 . hal-01289901

\section{HAL Id: hal-01289901 https://hal.science/hal-01289901}

Submitted on 17 Mar 2016

HAL is a multi-disciplinary open access archive for the deposit and dissemination of scientific research documents, whether they are published or not. The documents may come from teaching and research institutions in France or abroad, or from public or private research centers.
L'archive ouverte pluridisciplinaire HAL, est destinée au dépôt et à la diffusion de documents scientifiques de niveau recherche, publiés ou non, émanant des établissements d'enseignement et de recherche français ou étrangers, des laboratoires publics ou privés. 


\title{
Performance of a Digital Transmitter Leakage LMS-Based Cancellation Algorithm for Multi-Standard Radio-Frequency Transceivers ${ }^{\text {ts }}$
}

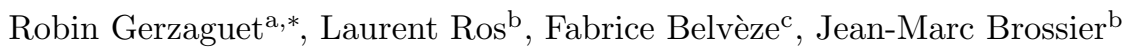 \\ ${ }^{a}$ CEA-Leti, Minatec Campus, Grenoble, France \\ ${ }^{b}$ GIPSA-Lab, Image and Signal Department, BP 46, 38402 Saint Martin d'Hères, France \\ ${ }^{c} S T$-Microelectronics, Grenoble, France
}

\begin{abstract}
This paper deals with a joint estimation algorithm that is dedicated to digital compensation of transmitter leakage pollution in frequency division duplexing transceivers. These transceivers are affected by transmitter to receiver signal leakage. Combined with the nonlinearity of amplifying components in the receiver path, the baseband signal received can be severely polluted by a baseband polluting term. This term is based on the square modulus of the transmitted signal, and it depends on the equivalent transmitter leakage channel which models leakages and the receiver path. Here, we consider a nonconvolutive, time-varying channel that is modeled by a time-varying complex gain and the presence of a fractional delay, modeling the propagation effects into the receiver. The complex gain is a sum of two components, a constant term that models static effects, and a first-order autoregressive model that approximates the time variation of the transmitter leakage channel. We focus here on a fully digital approach, using digital signal processing techniques and knowledge of the transmitted samples to mitigate the pollution. We first express the asymptotic performance of a transmitter leakage gain estimator piloted by a reference-based least-mean-square (LMS) approach in the synchronized case, and then we derive the influence of the fractional delay. We show that, in practice, the fractional delay cannot be neglected, and we propose a joint estimation of the fractional delay and the transmitter leakage gain to perform digital compensation. The proposed method is adaptive, recursive and online, and it has low complexity. This algorithm, that is developed for a flat transmitter leakage channel case, is seen to be robust in a typical selective channel simulation case, and more suitable than a classic multi-tap LMS scheme proposed in the literature.
\end{abstract}

Keywords: FDD transceiver; radio frequency impairment; Tx leakage; digital cancellation; least-mean-square (LMS) algorithm.

\section{Introduction}

In wireless communication systems, transmission and reception can be based on time division duplexing or on frequency division duplexing (FDD). In the first case, the transmission and the reception are temporally separated, leading to a time slot that is dedicated to transmission and another that is dedicated to reception [2]. However, for a FDD framework which is the case of the study presented here, the transmission and the reception are simultaneous, using two different carrier frequencies [3].

As a common antenna is used for both transmission and reception, a duplexer is used to connect the receiver $(\mathrm{Rx})$ path and the transmitter (Tx) path [4] (see Figure 1). In practice, as only an average of $50 \mathrm{~dB}$ attenuation can be

\footnotetext{
${ }^{\star}$ Part of this work was presented at the proceedings of the International Conference on Electronics Circuits and Systems [1]

* Corresponding author. Tel.:+33 38785279

Email addresses: robin.gerzaguet@cea.fr (Robin Gerzaguet),

laurent.ros@gipsa-lab.fr (Laurent Ros),

fabrice.belveze@st.com (Fabrice Belvèze),

jean-marc.brossier@gipsa-lab.fr (Jean-Marc Brossier)
}

achieved in the duplexer, the isolation between the $\mathrm{Tx}$ path and the Rx path is far from infinite [5]. Thus, the transmitted signal can leak into the Rx path, which leads to the so-called Tx leakage (TxL) phenomenon. As the uplink and the downlink bands are spectrally separated, the received signal will not be impaired directly by the leakage of the transmitted signal, as the low-pass filter (LPF) located after the demodulation stage will remove all of it from the band signals. However, an image of the transmitted signal can pollute the received signal in the baseband, due to nonlinearities and imperfections in the components in the Rx stage; especially the low-noise amplifier (LNA) [6] and the demodulator [7]. These aforementioned imperfections lead to second-order intermodulation products that shift downward to the baseband square component of the TxL signal [8]. In practical cases, this polluting signal can be neglected, except when the receiver is far from the transmitter (which corresponds to the cell edge context in cellular communications), as the power of the received signal is weak, and the power of the transmitted signal is strong [9].

To avoid this pollution, analog methods can be imple- 


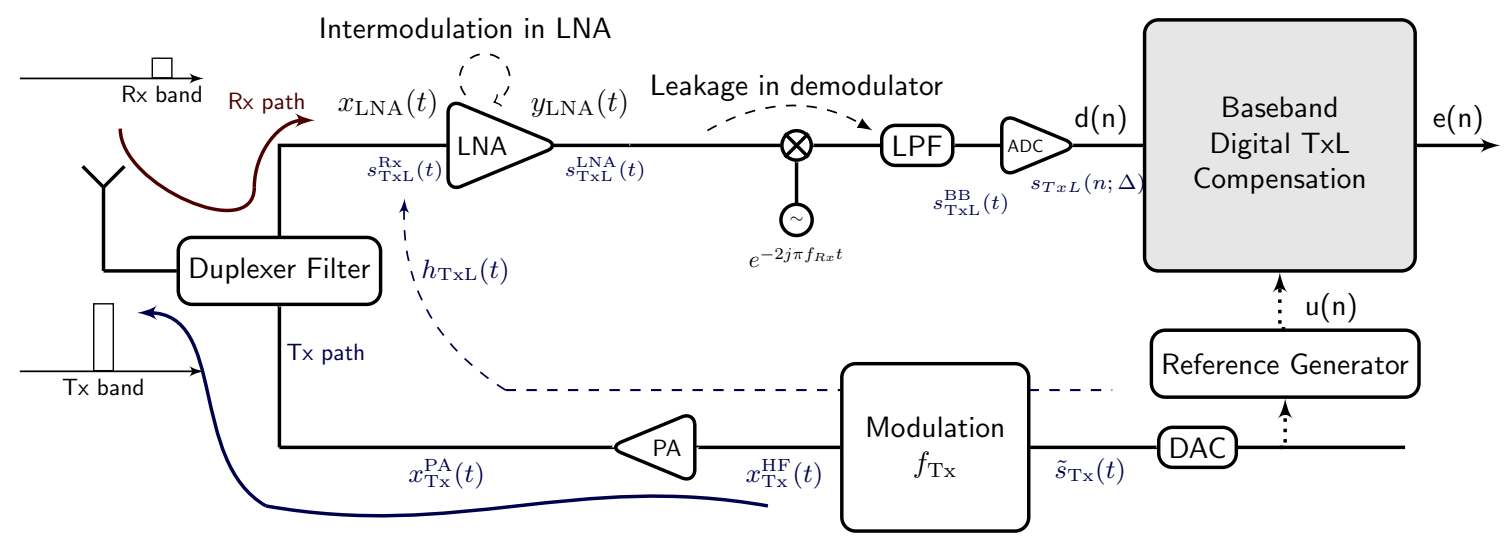

Figure 1: Classical frequency division duplexing chain in a radio-frequency (RF) transceiver with transmitter leakage baseband pollution. PA, power amplifier; ADC, analog-to-digital converter; DAC, digital-to-analog converter.

mented. Such mitigation methods consist of the addition of a band-pass filter in the Rx stage before [10] or after the LNA [11]. Other techniques consist of the combination of the Tx path multiplied by a calibrated analog gain to the Rx path, to suppress the pollution at high frequency [12]. However, RF transceivers contain more and more digital parts, and signal-processing techniques are becoming an area of interest for radio-frequency impairment problems [13]. In perfect agreement with the dirty $\mathrm{RF}$ paradigm, several digital-compensation methods have been investigated in the past few years for TxL compensation $[5,9,14,15]$.

These methods are based on the knowledge of the transmitted signal and require the estimation of the so-called TxL channel that characterizes the Tx-Rx isolation of the duplexer and the Rx path. Different channel models are used in the literature, from a single constant coefficient $[5,16]$, to an exponential decay filter [17], or a more general finite impulse response filter [9]. However, literature methods do not take into account that the duplexer isolation is time varying, as showed by [18]. Furthermore, under the flat TxL channel assumption, the existence of a fractional delay in the reception stage due to digital blocks, analog blocks and propagation into the receiver, has not been considered. Also, a steady-state analysis of a LMSbased algorithm was described by [19] to reduce the impact of the spurs (which are narrowband pollutions due to leakage of clock harmonics), and a similar approach can be investigated for a wideband polluting signal.

In this paper, we propose a digital compensation scheme for the TxL problem. Our method exploits the knowledge of the transmitted signal as a reference, and estimates both the TxL channel that is assumed to be frequency flat and to follow a first-order auto-regressive model (AR1), and the fractional delay introduced by the transceiver. We first study the synchronized case (where the fractional delay is assumed to be perfectly known), and we derive the asymptotic performance of the channel complex gain estimator, based on the LMS algorithm. Then, through the derivation of the analytic formula of the asymptotic performance of the algorithm in the presence of noncompensated fractional delay, we show that the fractional delay must be compensated for beforehand. We propose a joint estimation algorithm for the estimation of both the channel gain and the fractional delay. Before the compensation part, the estimation part of our algorithm has similitudes with a data-aided algorithm that was designed for phase and timing synchronization as well as for automatic gain control [20], although here the Rx signal is considered as noise for the estimation process. To be suitable for hardware implementation, this algorithm is adaptive, online, and recursive, and has low complexity. The algorithm is proposed under the flat TxL channel assumption, but we will see in the simulation part that the method is robust against a typical frequency-selective TxL channel, and is more suitable than a classical multi-tap LMS approach when the fractional delay is not negligible. We complete and extend the initial work proposed by [1], providing here all of the proofs, additional extensive simulations, comparisons with the literature, and interpretations. The main contributions of the present paper can be summarized as:

- Proposition of a baseband digital model for TxL pollution, which takes into account the time variation of the polluting channel and the propagation delay to the receiver. The link between the parameters of the (AR1-based) model and physical values (e.g., interference level, normalized Doppler frequency, and others) is analytically established.

- Proposition of a joint estimation algorithm to both estimate the time-varying complex gain and the fractional delay, before performing TxL compensation. This LMS-based compensation is piloted by a reference synthesized from the transmitted samples.

- Derivation of the closed-form asymptotic performance of the algorithm when the fractional delay is assumed to be perfectly known. This can be used to tune 
the step-size parameters of the algorithm, as well as to predict the signal interference ratio (SIR) performance with respect to the TxL channel properties. The impact of a noncompensated fractional delay on the asymptotic performance is also derived, which shows that the fractional delay must be compensated for beforehand.

- Simulation and comparison between the proposed joint estimation scheme and a classical literature method for the case of a frequency selective TxL channel, which shows the robustness and effectiveness of the proposed algorithm.

This paper is organized as follows. We give the baseband polluting model in Section 2. We derive the asymptotic performance of the LMS algorithm for the channel gain estimation process in the synchronized case in Section 3. We derive the analytic formulae of the influence of the fractional delay in Section 4. We propose a joint channel gain and fractional delay in Section 5. Section 6 validates our method and theoretical results through simulations.

\section{System model}

\subsection{Pollution model}

As, the baseband polluting model involves several signals from both Tx and Rx stages, the main signals (and their definitions) are synthesized in table 1, and also mentioned in Figure 1. We consider a classical FDD scheme where transmission and reception are simultaneous, at the frequency $f_{\mathrm{Tx}}$ for transmission, and $f_{\mathrm{Rx}}$ for reception. The transmitted signal $x_{\mathrm{Tx}}^{\mathrm{HF}}(t)$ has a bandwidth $B_{T x}$ around $f_{\mathrm{Tx}}$ (i.e the bandwidth of $x_{\mathrm{Tx}}^{\mathrm{HF}}(t)$ is from $f_{\mathrm{Tx}}-B_{T x} / 2$ to $\left.f_{\mathrm{Tx}}+B_{T x} / 2\right)$, and can be expressed as:

$$
x_{\mathrm{Tx}}^{\mathrm{HF}}(t)=\Re\left\{\tilde{s}_{\mathrm{Tx}}(t) e^{2 j \pi f_{\mathrm{Tx}} t+j \phi_{\mathrm{Tx}}}\right\},
$$

where $\Re\{\}$ is the real part operator, and where:

$$
\tilde{s}_{\mathrm{Tx}}(t)=s_{\mathrm{Tx}_{\mathrm{I}}}(t)+j s_{\mathrm{Tx}_{Q}}(t),
$$

is the complex baseband Tx signal. Here, we assume a purely linear power amplifier with gain $g_{\mathrm{PA}}$, and the signal at the duplexer input can be expressed as

$$
x_{\mathrm{Tx}}^{\mathrm{PA}}(t)=g_{\mathrm{PA}} \times x_{\mathrm{Tx}}^{\mathrm{HF}}(t) .
$$

In a classical FDD transceiver, TxL is due to the cascade of several impairments:

- The finite isolation between the Tx path and Rx path at the duplexer implies leakage of the transmitted signal into the Rx path (see Figure 1). If we denote $h_{\mathrm{TxL}}(t)$ as the duplexer equivalent channel, and introduce the power amplifier gain $g_{\mathrm{PA}}$ into the

\section{Analog signals}

\begin{tabular}{c|c}
$\tilde{s}_{\mathrm{Tx}}(t)$ & Complex baseband transmitted signal \\
$x_{\mathrm{Tx}}^{\mathrm{HF}}(t)$ & Transmitted signal at $f_{\mathrm{Tx}}$ \\
$x_{\mathrm{Tx}}^{\mathrm{PA}}(t)$ & Transmitted signal after amplification \\
$s_{\mathrm{TxL}}^{\mathrm{HF}}(t)$ & Leaked transmitted signal in receiver path \\
$h_{\mathrm{TxL}}(t)$ & Duplexer leakage equivalent channel \\
$x_{\mathrm{LNA}}(t)$ & Signal in Rx stage at input of LNA \\
$y_{\mathrm{LNA}}(t)$ & Signal in Rx stage at output of LNA \\
$s_{\mathrm{TxL}}^{\mathrm{BB}}(t)$ & Tx leakage baseband polluting term \\
& Baseband digital signals
\end{tabular}

$s_{T x L}(n ; \Delta) \mid$ Sampled Tx leakage baseband polluting term $d(n)$

$\beta_{\mathrm{TxL}}(n)$

$\alpha_{\mathrm{TxL}}(n)$

$\xi_{\mathrm{TxL}}(n)$

$u(n)$

$u_{\delta}(n)$

$e(n)$

Discrete time observation signal

Complex TxL gain

Time varying part of complex gain

State noise of time varying gain

Reference signal for compensation

Fractionally delayed reference

Signal after compensation

Table 1: List of most important signals

duplexer response, the TxL signal in the $\mathrm{Rx}$ path can be expressed as

$$
s_{\mathrm{TxL}}^{\mathrm{HF}}(t)=h_{\mathrm{TxL}}(t) * x_{\mathrm{Tx}}^{\mathrm{HF}}(t)
$$

where $*$ is the convolution operator. The signal at the input of the LNA $x_{\mathrm{LNA}}(t)$ is a mixture between the desired high-frequency signal $s_{\mathrm{Rx}}^{\mathrm{HF}}(t)$ and the TxL signal $s_{\mathrm{TxL}}^{\mathrm{HF}}(t)$. It can be noted that, in practice, the level of the received signal $s_{\mathrm{Rx}}^{\mathrm{HF}}(t)$ can be much lower than the level of the leaked transmitted signal $s_{\mathrm{TxL}}^{\mathrm{HF}}(t)$, especially in a cell edge.

$$
x_{\mathrm{LNA}}(t)=s_{\mathrm{Rx}}^{\mathrm{HF}}(t)+s_{\mathrm{TxL}}^{\mathrm{HF}}(t)
$$

- The LNA that amplifies the received signal regarding the noise is not perfectly linear [21]. As a consequence, intermodulation products located at the LNA create second-order baseband components. Assuming a LNA with a nonlinearity of order II, the output of the LNA can be expressed as:

$$
y_{\mathrm{LNA}}(t)=\alpha_{1}^{\mathrm{LNA}} x_{\mathrm{LNA}}(t)+\alpha_{2}^{\mathrm{LNA}} x_{\mathrm{LNA}}^{2}(t)
$$

By introducing $z_{\mathrm{Rx}}(t)$ as the analytic signal associated with the received signal $s_{\mathrm{Rx}}^{\mathrm{HF}}(t)$ [and with a baseband complex envelop $\left.\tilde{s}_{\mathrm{Rx}}(t)\right]$, and $z_{\mathrm{TxL}}(t)$ as the analytic signal of $s_{\mathrm{TxL}}^{\mathrm{HF}}(t)$ [with a baseband complex envelop $\left.\tilde{s}_{\mathrm{TxL}}(t)\right]$, defined as:

$$
\begin{aligned}
z_{\mathrm{Rx}}(t) & =s_{\mathrm{Rx}}(t) e^{2 j \pi f_{\mathrm{Rx}} t} \\
z_{\mathrm{TxL}}(t) & =\tilde{s}_{\mathrm{TxL}}(t) e^{2 j \pi f_{\mathrm{Tx}} t}
\end{aligned}
$$


the link with the high-frequency signals can be expressed as:

$$
\begin{aligned}
s_{\mathrm{Rx}}^{\mathrm{HF}}(t) & =\frac{z_{\mathrm{Rx}}(t)+z_{\mathrm{Rx}}^{*}(t)}{2}=\Re\left\{z_{\mathrm{Rx}}(t)\right\} \\
s_{\mathrm{TxL}}^{\mathrm{HF}}(t) & =\frac{z_{\mathrm{TxL}}(t)+z_{\mathrm{TxL}}^{*}(t)}{2}=\Re\left\{z_{\mathrm{TxL}}(t)\right\},
\end{aligned}
$$

which after amplification, leads to:

$$
\begin{aligned}
y_{\mathrm{LNA}}(t)=\alpha_{1}^{\mathrm{LNA}}\left[s_{\mathrm{Rx}}^{\mathrm{HF}}(t)+s_{\mathrm{TxL}}^{\mathrm{HF}}(t)\right] \\
+\frac{\alpha_{2}^{\mathrm{LNA}}}{4}[\underbrace{z_{\mathrm{TxL}}(t)^{2}}_{2 \mathrm{f}_{\mathrm{Tx}}}+\underbrace{z_{\mathrm{TxL}}^{*}(t)^{2}}_{-2 f_{\mathrm{Tx}}}+\underbrace{z_{\mathrm{Rx}}(t)^{2}}_{2 f_{\mathrm{Rx}}}+\underbrace{z_{\mathrm{Rx}}^{*}(t)^{2}}_{-2 f_{\mathrm{Rx}}}] \\
+\frac{\alpha_{2}^{\mathrm{LNA}}}{2}[\underbrace{z_{\mathrm{TxL}}(t) z_{\mathrm{TxL}}^{*}(t)}_{\text {Baseband }}+\underbrace{z_{\mathrm{TxL}}(t) z_{\mathrm{Rx}}(t)}_{f_{\mathrm{Tx}}+f_{\mathrm{Rx}}}+\underbrace{z_{\mathrm{TxL}}^{*}(t) z_{\mathrm{Rx}}^{*}(t)}_{-f_{\mathrm{Tx}}-f_{\mathrm{Rx}}}] \\
+\frac{\alpha_{2}^{\mathrm{LNA}}}{2}[\underbrace{z_{\mathrm{Rx}}(t) z_{\mathrm{Rx}}^{*}(t)}_{\text {Baseband }}+\underbrace{z_{\mathrm{TxL}}^{*}(t) z_{\mathrm{Rx}}(t)}_{-f_{\mathrm{Tx}}+f_{\mathrm{Rx}}}+\underbrace{z_{\mathrm{TxL}}(t) z_{\mathrm{Rx}}^{*}(t)}_{f_{\mathrm{Tx}}-f_{\mathrm{Rx}}}] .
\end{aligned}
$$

whereby the baseband term relative to the TxL pollution is finally:

$$
s_{\mathrm{TxL}}^{\mathrm{Rx}}(t)=\frac{\alpha_{2}^{\mathrm{LNA}}}{2}\left|\tilde{s}_{\mathrm{TxL}}(t)\right|^{2} .
$$

Again, as the level of the received signal is low, the baseband polluting term due to the square product of the received signal is negligible compared to $s_{\mathrm{TxL}}^{\mathrm{Rx}}(t)$ :

- A leakage can appear at the demodulator stage, between the input and the output of the demodulator [6]. As a consequence, baseband signals (due to intermodulation products) will pollute the desired noisy signal that is translated from $f_{\mathrm{Rx}}$ to baseband by the demodulator. If we denote $g_{\mathrm{OL}}$ as the static gain of the leakage, and $h_{\mathrm{Rx}}(t)$ as the low-pass filter located after the demodulation stage, the final baseband polluting term is:

$$
s_{\mathrm{TxL}}^{\mathrm{BB}}(t)=g_{\mathrm{OL}} \times \frac{\alpha_{2}^{\mathrm{LNA}}}{2} \times h_{\mathrm{Rx}}(t) *\left|\tilde{s}_{\mathrm{TxL}}(t)\right|^{2}
$$

It can be noted that the TxL pollution process can be explained from different angles, with the same final baseband pollution model: e.g., with a perfectly linear LNA, intermodulation products can appear in the $\mathrm{Rx}$ baseband stage due to leakage between the input of the demodulation stage and the signal used for demodulation, as described by [9].

Here, as we will apply digital compensation to mitigate the impact of the TxL pollution, we need a digital pollution model for the TxL. The polluting term after the analog-to-digital converter can be expressed as:

$$
s_{T x L}(n)=\left[s_{\mathrm{TxL}}^{\mathrm{BB}}(t)\right]_{t=n T_{R x}}
$$

where $T_{\mathrm{Rx}}=1 / F_{\mathrm{Rx}}$ is the sampling period. Here, for the theoretical analysis, we assume that the duplexer is frequency flat, and that the Rx chain introduces a complex gain due to mismatch, as has been widely assumed in the literature, as in $[5,16,22]$.

Due to digital and analog blocks, the polluting signal has a delay $\Delta=(D+\delta) T_{\mathrm{Rx}}$, which is composed of an integer part $D$ and a fractional part $\delta$. From (9) and (10), the polluting term becomes:

$s_{T x L}(n ; \Delta)=\beta_{\mathrm{TxL}}(n) \times\left[h_{\mathrm{Rx}}(t-\Delta) *\left|\tilde{s}_{T x}(t-\Delta)\right|^{2}\right]_{t=n T_{R x}}$

where $\beta_{\mathrm{TxL}}(n)$ models the global TxL complex channel gain at time index $n$ (modeling the impact of both the duplexer, the Rx chain, and the demodulator). The TxL channel gain is time varying [18], and can be expressed as:

$$
\beta_{\mathrm{TxL}}(n)=\beta_{0_{\mathrm{TxL}}}+\alpha_{\mathrm{TxL}}(n),
$$

where $\beta_{0_{\mathrm{TxL}}}$ is a complex constant that is linked to the interference level, and $\alpha_{\mathrm{TxL}}(n)$ is the time-varying channel evolution. For the complex gain evolution, we use a firstorder auto-regressive process that is a simple but widely used model in the communications literature used to approximate time-varying channels [23, 24], and is defined by:

$$
\alpha_{\mathrm{TxL}}(n)=\gamma \alpha_{\mathrm{TxL}}(n-1)+\xi_{\mathrm{TxL}}(n) .
$$

initialized by $\alpha_{\mathrm{TxL}}(0)=0$, where $0<\gamma<1$ and $\xi_{\mathrm{TxL}}(n)$ is circular complex zero-mean white Gaussian state noise, with variance:

$$
\sigma_{\xi_{\mathrm{TxL}}}^{2}=\left(1-\gamma^{2}\right) \sigma_{\alpha_{\mathrm{TxL}}}^{2} .
$$

As the channel is composed of a static term and a complex Gaussian dynamic term, we denote $K$ as the power ratio of the fixed and scattered components (in the same way as for a Ricean wireless channel model):

$$
K=\frac{\left|\beta_{0_{\mathrm{T \times L}}}\right|^{2}}{\sigma_{\alpha_{\mathrm{T} \times \mathrm{L}}}^{2}}
$$

The case $K \rightarrow \infty$ corresponds to a nonvarying channel, whereas $K \rightarrow 0$ corresponds to a varying channel with a negligible constant interference level.

\subsection{Observation model and estimation objectives}

We assume that the integer part of the delay is known, as in practice it can be easily estimated with a correlation process or with a parallel structure, as described by [5], and without loss of generality, we take $D=0$. In the baseband receiver part, the discrete time observed signal $d(n)$ becomes:

$$
d(n)=x(n)+b(n)+s_{T x L}(n ; \delta),
$$

where $x(n)$ is the desired signal, which is assumed to be stationary uncorrelated ${ }^{1}$ and zero-mean, of variance $\sigma_{x}^{2}$,

\footnotetext{
${ }^{1}$ This strong hypothesis is only made to derive theoretical performance formulae, but we will see in the simulation section that the results can be applied to some typical communication signals (that are cyclostationary and slightly correlated by the waveforms)
} 
$b(n)$ is the white additive Gaussian noise, of variance $\sigma_{b}^{2}$, and $s_{T x L}(n ; \delta)$ is the TxL polluting signal defined in (11).

The TxL canceller is not intended to cancel the additive noise term. As we focus here on the performance of the TxL canceller, and not on the impact of the additive white noise, we consider for the performance measurement that the noise is part of the desired signal. In other words, regarding the TxL cancellation algorithm, the desired signal is the observed signal without the TxL polluting term $s_{T x L}(n ; \delta)$. We denote $x_{b}(n)$ as the desired noisy signal, which is a white zero-mean signal, of variance $\sigma_{x_{b}}^{2}$, and the observation model becomes:

$$
d(n)=x_{b}(n)+s_{T x L}(n ; \delta)
$$

The influence of the TxL in terms of the SIR, expressed in $\mathrm{dB}$, is:

$$
\mathrm{SIR}_{\text {init }}=-10 \log _{10}\left(\frac{P_{s_{T x L}}}{\sigma_{x_{b}}^{2}}\right),
$$

where $P_{s_{T x L}}$ is the power of the polluting term $s_{T x L}(n ; \delta)$.

To proceed to the compensation, we synthesize a reference $u(n)$ as a perfect image of the baseband intermodulation product that takes into account the impact of the Rx chain:

$$
u(n)=\left[h_{\mathrm{Rx}}(t) *\left|s_{T x}(t)\right|^{2}\right]_{t=n T_{R x}}
$$

This reference of power $P_{u}$ is synthesized in the reference generator (see Figure 1) from the baseband Tx samples, and it can be noted that in practice, it might need additional processing, such as an upsampler, filters, and a resampler. Based on the observation model described in (17), the TxL channel evolution described in (12) and (13), and the reference in (19), the estimation process consists of the estimation of the time-varying complex gain $\beta_{\mathrm{TxL}}(n)$ and the fractional delay $\delta$.

The estimation error is defined by:

$$
\epsilon(n)=e(n)-x_{b}(n),
$$

where $e(n)$ is the output of the TxL compensation structure (see Figure 1), and the asymptotic performance of the algorithm will be described by the SIR after compensation:

$$
\mathrm{SIR}_{\text {comp }}=-10 \log _{10}\left(\frac{P_{\epsilon}}{\sigma_{x_{b}}^{2}}\right) .
$$

where $P_{\epsilon}$ is the mean square error.

\subsection{Link between coherence time and AR1 parameters}

The baseband model of the complex gain $\beta_{\mathrm{TxL}}(n)$ is function of several parameters, synthesized in table 2 . In addition to the overall parameters, that are necessary to establish the model and to derive the analytical study, the model also depends on the so-called primary parameters that characterizes the AR1 mathematical model (see table 2). Besides, instead of using the primary parameters, physical interpretation of the baseband polluting model

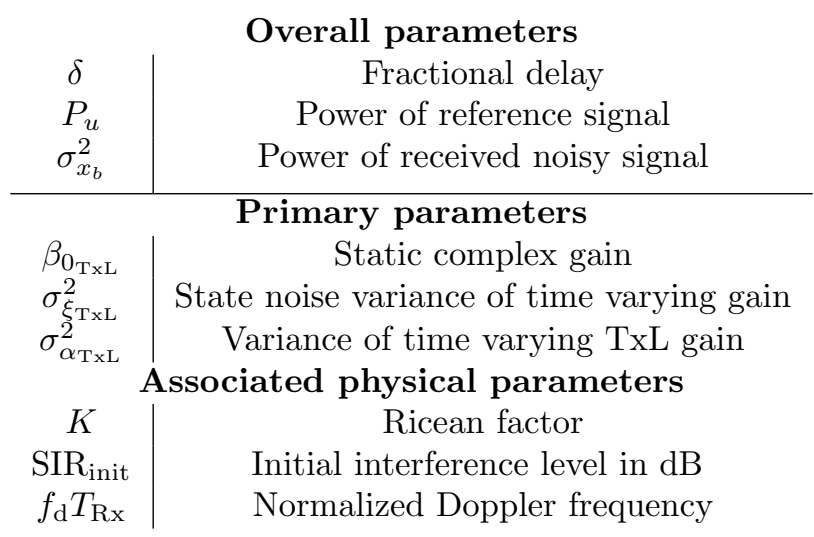

Table 2: List of parameters

and characterization of the parameters can alternatively be done through the initial SIR (that defines the interference level at the entry of the digital front end), the ratio $K$, and the normalized Doppler frequency $f_{\mathrm{d}} T_{\mathrm{Rx}}$. These parameters are denoted as associated physical parameters in table 2 .

The output of the AR1 model can be viewed as the low-pass filtering of the state noise $\xi_{\mathrm{TxL}}(n)$, and we denote $L(z)$ as the $\mathrm{z}$ transform of this filter. From (13), we have:

$$
L(z)=\frac{1}{1-\gamma z^{-1}}
$$

and the coherence time $T_{\text {corr }}$, defined here as the inverse of the 3-dB cut-off frequency of $L(z)$, can be approximated as:

$$
T_{\mathrm{corr}} \approx \frac{2 \gamma \pi T_{\mathrm{Rx}}}{1-\gamma}
$$

The channel variation is slow in terms of the Rx sampling time $T_{\mathrm{Rx}}$, and we assume $T_{\text {corr }} \gg T_{\mathrm{Rx}}$; the AR1 parameter $\gamma$ can be approximated as:

$$
\gamma \approx \frac{T_{\text {corr }}}{T_{\text {corr }}+2 \pi T_{\mathrm{Rx}}} .
$$

Reintroducing (24) in (14), and introducing the normalized Doppler frequency $f_{\mathrm{d}} T_{\mathrm{Rx}}=\frac{T_{\mathrm{Rx}}}{T_{\text {corr }}} \ll 1$, we finally have:

$$
\sigma_{\xi_{\mathrm{T} \times \mathrm{L}}}^{2} \approx 4 \pi \sigma_{\alpha_{\mathrm{T} \times \mathrm{L}}}^{2} f_{\mathrm{d}} T_{\mathrm{Rx}}
$$

and finally, the constant interference level $\beta_{0_{\mathrm{T} \times \mathrm{L}}}$ and the channel variance $\sigma_{\alpha_{\mathrm{Tx}}}^{2}$ are defined by the initial SIR and the ratio $K$ :

$$
\begin{aligned}
\left|\beta_{0_{\mathrm{T} \times \mathrm{L}}}\right|^{2} & =\frac{\sigma_{x_{b}}^{2}}{2 P_{u}} \frac{K}{1+K} 10^{-\mathrm{SIR}_{\text {init }}} \\
\sigma_{\alpha_{\mathrm{T} \times \mathrm{L}}}^{2} & =\frac{\left|\beta_{0_{\mathrm{TxL}}}\right|^{2}}{K} .
\end{aligned}
$$

As in classical literature articles related to channel estimation modeled by auto-regressive models, the theoretical performance formulae in the rest of the paper are expressed in terms of $\beta_{0_{\mathrm{Tx} \mathrm{L}}}, \sigma_{\xi_{\mathrm{T} x \mathrm{~L}}}^{2}$ and $\sigma_{\alpha_{\mathrm{T} x \mathrm{~L}}}^{2}$ that define the baseband 
polluting model described in (12)-(15). It corresponds to the primary parameters described in Table 2 that can be associated to practical physical parameters (see Table 2) with (25)-(27).

\section{Complex gain estimator and performance in the synchronized case}

In this part, we assume that $\delta$ is known and we synthesize the fractionally delayed reference $u_{\delta}(n)$ from $(19)$ :

$$
u_{\delta}(n)=\left[h_{\mathrm{Rx}}(t) *\left|s_{T x}(t)\right|^{2}\right]_{t=n T_{R x}+\delta} .
$$

Note that with a perfect interpolator, we would have:

$$
s_{\mathrm{TxL}}(n, \delta)=\beta_{\mathrm{TxL}}(n) u_{\delta}(n),
$$

and it can also be noted that in practical cases, a Lagrangian interpolator of size $L$ can be implemented [25, 26]. The compensation idea, as depicted in Figure 2, is to obtain the compensated signal $e(n)$ by subtracting the estimated TxL component from the input sample $d(n)$. This component is obtained by multiplying the reference signal $u_{\delta}(n)$ by a complex adaptive coefficient $\hat{\beta}_{T x L}(n)$ (estimation of the TxL channel), which is updated using $e(n)$ with a LMS algorithm [27]:

$$
\begin{aligned}
\hat{s}_{\mathrm{TxL}}(n) & =\hat{\beta}_{\mathrm{TxL}}(n) u_{\delta}(n) \\
e(n) & =d(n)-\hat{s}_{\mathrm{TxL}}(n) \\
\hat{\beta}_{\mathrm{TxL}}(n+1) & =\hat{\beta}_{\mathrm{TxL}}(n)+\mu u_{\delta}(n) e(n),
\end{aligned}
$$

where $\mu$ is the constant step size of the algorithm. The asymptotic performance of the algorithm can be derived as (see Appendix A):

$$
\mathrm{SIR}_{\text {comp }}=-10 \log _{10}\left[\frac{\mu P_{u}}{2-\mu P_{u}}+\frac{Q\left(P_{u}, \sigma_{\alpha_{\mathrm{T} \times \mathrm{L}}}^{2}, \gamma\right)}{\mu \sigma_{x_{b}}^{2}\left(2-\mu P_{u}\right)}\right]
$$

where

$$
Q\left(P_{u}, \sigma_{\alpha_{\mathrm{T} \times \mathrm{L}}}^{2}, \gamma\right)=\frac{2 \mu P_{u} \sigma_{\alpha_{\mathrm{T \times L}}}^{2}(1-\gamma)}{1-\gamma\left(1-\mu P_{u}\right)}
$$

models the impact of channel variations on performance. It can be noted that the asymptotic performance has a form that is similar to the performance of the spur canceller in [19]. The asymptotic SIR is composed of a static term (due to the presence of the input signal $x_{b}(n)$ ) and a dynamic term (34) due to channel variations. The global form of this asymptotic SIR is linked to the stochastic gradient approach, and the difference lies in the use of different pollution models.

From the previous result, an optimal functional point can be deduced. First, with (14), we can relate $\gamma$ to $\sigma_{\xi_{\mathrm{T} \times \mathrm{L}}}^{2}$ and $\sigma_{\alpha_{\mathrm{TxL}}}^{2}$ :

$$
\gamma=\sqrt{1-\frac{\sigma_{\xi_{\mathrm{T} \times \mathrm{L}}}^{2}}{\sigma_{\alpha_{\mathrm{T} \times \mathrm{L}}}^{2}}}
$$

with $\gamma \approx 1$ as we assume $\sigma_{\xi_{\mathrm{TxL}}}^{2} \ll \sigma_{\alpha_{\mathrm{TxL}}}^{2}$. If we additionally assume that $\mu P_{u} \ll 1$, the SIR can be approximated by:

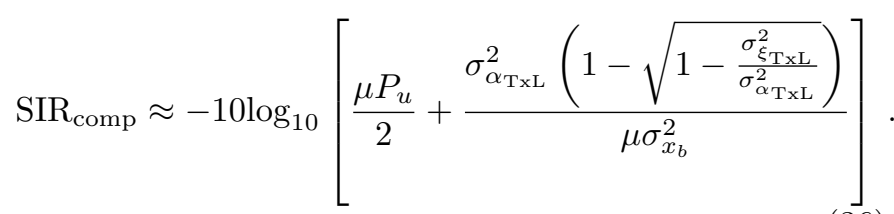

From (36), an optimal step size can be deduced:

$$
\mu_{\mathrm{opt}}=\sqrt{\frac{2 \sigma_{\alpha_{\mathrm{TxL}}}^{2}}{P_{u} \sigma_{x_{b}}^{2}}\left(1-\sqrt{\left.1-\frac{\sigma_{\xi_{\mathrm{TxL}}}^{2}}{\sigma_{\alpha_{\mathrm{T} \times \mathrm{L}}}^{2}}\right)}\right.}
$$

and reintroducing (37) in (36) leads to the maximal SIR reachable for a given AR1 channel modeling the TxL impact:

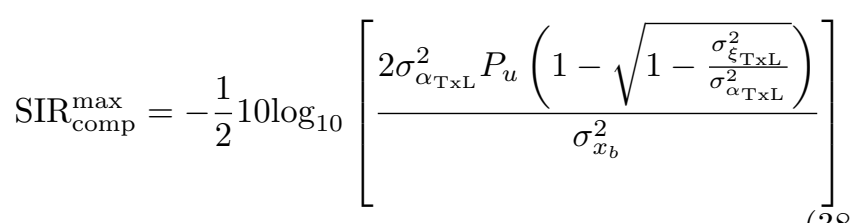

\section{Influence of fractional delay}

In the previous section, we assumed that the fractional delay $\delta$ was perfectly known. In this section, we study the impact of a non-compensated fractional delay on the performance of the complex gain estimator. If we use in the algorithm a reference that is not fractionally delayed, the estimation process of the polluting term $\hat{s}_{\mathrm{TxL}}(n)$ would be $\hat{\beta}_{\mathrm{TxL}}(n) u(n)$ [with $u(n)$ defined in (19)], and not $\hat{\beta}_{\mathrm{TxL}}(n) u_{\delta}(n)[$ as in $(30)]$.

Thus, the reference used in the algorithm would not longer be a perfect image of the polluting term (see (29)) and in this case, the LMS algorithm becomes:

$$
\begin{aligned}
\hat{s}_{\mathrm{TxL}}(n) & =\hat{\beta}_{\mathrm{TxL}}(n) u(n) \\
e(n) & =\left[x_{b}(n)+\beta_{\mathrm{TxL}}(n) u_{\delta}(n)\right]-\hat{s}_{\mathrm{TxL}}(n) \\
\hat{\beta}_{\mathrm{TxL}}(n+1) & =\hat{\beta}_{\mathrm{TxL}}(n)+\mu u(n) e(n),
\end{aligned}
$$

and we introduce:

$$
\eta_{\mathrm{TxL}}(n)=u_{\delta}(n)-u(n)
$$

as the difference between the perfect reference and the used reference. We assume that $\eta_{\mathrm{TxL}}(n)$ can be modeled as white Gaussian noise, of variance $\sigma_{\eta_{\mathrm{T} \times \mathrm{L}}}^{2}$. On a first approximation (assuming linear interpolation), the variance of $\eta_{\text {TxL }}$ can be expressed as (see Appendix B):

$$
\sigma_{\eta_{\mathrm{Tx \textrm {L }}}}^{2} \approx \delta^{2} P_{u}
$$

In the case of a non-compensated fractional delay, the asymptotic performance of the system is a function of the 


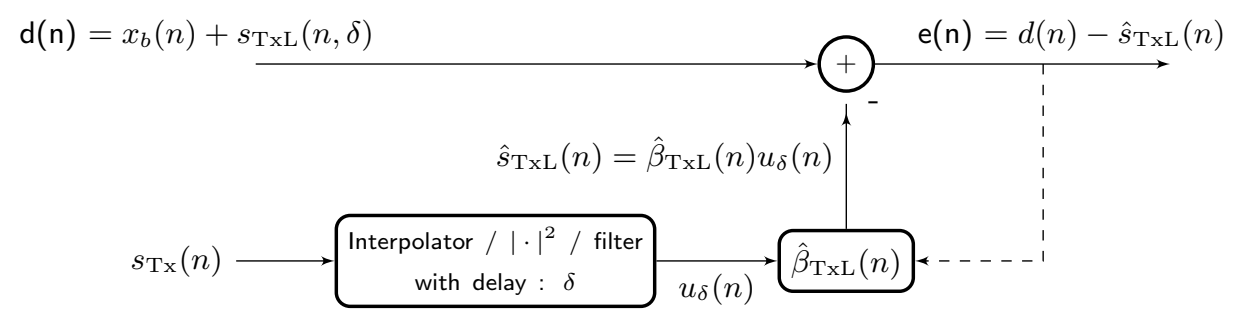

Figure 2: Gain estimation in the case of a known fractional delay.

AR1 parameters and the variance of the noise introduced in (39), and it can be expressed as (see Appendix C):

$$
\begin{aligned}
& \operatorname{SIR}_{\mathrm{comp}} \approx-10 \log _{10}\left[\frac{\mu P_{u}}{2-\mu P_{u}}+\frac{Q\left(P_{u}, \sigma_{\alpha_{\mathrm{TxL}}}^{2}, \gamma\right)}{\mu\left(\sigma_{x}^{2}+\sigma_{b}^{2}\right)\left(2-\mu P_{u}\right)}\right. \\
& \left.+\frac{\left.\left.\mu P_{u}\left(\mid \beta_{0_{\mathrm{T} \times \mathrm{L}}}\right)\right|^{2}+\sigma_{\alpha_{\mathrm{TxL}}}^{2}\right) \sigma_{\eta_{\mathrm{TxL}}}^{2}}{\sigma_{x_{b}}^{2}\left(2-\mu P_{u}\right)}+\left(\left|\beta_{0_{\mathrm{T} \times \mathrm{L}}}\right|^{2}+\sigma_{\alpha_{\mathrm{T} \times \mathrm{L}}}^{2}\right) \frac{\sigma_{\eta_{\mathrm{TxL}}}^{2}}{\sigma_{x_{b}}^{2}}\right]
\end{aligned}
$$

The asymptotic SIR is composed of four terms: the first and the second are relative to the synchronized LMS performance, and linked to the static error and the dynamic error (due to the AR1 channel), and the two last are due to the non-compensation of the fractional delay. It can be noted that the fractional delay has a non-negligible impact on the asymptotic performance, and it must be compensated for upstream. As $\delta$ can be unknown in practice, a joint estimation scheme must be investigated.

\section{Joint estimation algorithm}

To jointly estimate the complex channel gain $\beta_{\mathrm{TxL}}(n)$ and the fractional delay $\delta$, we propose the joint estimation process depicted in Figure (3).

The reference $u_{\delta_{n}}(n)$ used in the gain estimator algorithm defined in (31)-(32) is obtained from $u(n)$, which is delayed with a fractional delay $\delta_{n}$, with $(28)$. Then, using the output $e(n)$ of the gain estimator block, the channel estimate $\hat{\beta}_{\mathrm{TxL}}(n)$, the reference $u(n)$, and the fractionally delayed reference $u_{\delta_{n}}(n)$, the update of the fractional delay estimate $\delta_{n+1}$ is performed with another LMS algorithm. The updated fractional delay estimate $\delta_{n+1}$ is then used in the next iteration of the compensation scheme.

The fractional delay estimation algorithm is based on the minimisation of the instantaneous square error $|e(n)|^{2}$ to which a gradient is applied (see Appendix D):

$$
\begin{aligned}
\Delta_{u}(n)= & u(n-1)-u(n) \\
\delta_{n+1}= & \delta_{n}+\nu \Re\left\{\left[\hat{\beta}_{T x L}(n) \Delta_{u}(n)\right.\right. \\
& \left.\left.+u_{\delta_{n}}(n) L(n)\right] e^{*}(n)\right\} \\
L(n+1)= & \left(1-\mu u_{\delta_{n}}(n)^{2}\right) L(n)-\mu d(n) \Delta_{u}(n) \\
+ & 2 \mu \hat{\beta}_{\mathrm{TxL}}(n) u_{\delta_{n}}(n) \Delta_{u}(n),
\end{aligned}
$$

where $L(n)=\partial \hat{\beta}_{\mathrm{TxL}}(n) / \partial \delta$ and $\nu$, the constant step size of the fractional delay estimator. In practice, $\nu$ can be chosen in a wide range, but must be normalized by the power of the signal $\sigma_{x_{b}}^{2}$.

The final joint estimation algorithm is composed of (31)(32), (42)-(44), and it can be expressed as:

$$
\begin{aligned}
e(n)= & d(n)-\hat{\beta}_{\mathrm{TxL}}(n) u_{\delta_{n}}(n) \\
\hat{\beta}_{\mathrm{TxL}}(n+1)= & \hat{\beta}_{\mathrm{TxL}}(n)+\mu u_{\delta_{n}}(n) e(n) \\
\Delta_{u}(n)= & u(n-1)-u(n) \\
\delta_{n+1}= & \delta_{n}+\nu \Re\left\{\left[\hat{\beta}_{\mathrm{TxL}}(n) \Delta_{u}(n)+\right.\right. \\
& \left.\left.u_{\delta_{n}}(n) L(n)\right] e^{*}(n)\right\} \\
L(n+1)= & \left(1-\mu u_{\delta_{n}}(n)^{2}\right) L(n)-\mu d(n) \Delta_{u}(n) \\
& +2 \mu \hat{\beta}_{\mathrm{TxL}}(n) u_{\delta_{n}}(n) \Delta_{u}(n) .
\end{aligned}
$$

It can be seen that this algorithm is recursive, online (as it provides a compensated output $e(n)$ at each iteration), and with low complexity. A computational complexity comparison is done at the end of Section 6 .

\section{Simulations}

The performance of the proposed algorithm, and the influence of both channel variations and constant fractional delay are further analyzed by simulations. In this section, the results are presented with respect to the physical parameters given in table 2 ( i.e the normalized Doppler frequency $f_{\mathrm{d}} T_{\mathrm{Rx}}$, the initial SIR and the Ricean factor $K$ ). The links between the primary set of parameters (the variance of the time varying channel $\sigma_{\alpha_{\mathrm{T} \times \mathrm{L}}}^{2}$, the constant interference level $\beta_{0_{\mathrm{TxL}}}$ and the speed of the AR1 process $\sigma_{\xi_{\mathrm{T} \times \mathrm{L}}}^{2}$ ) and the physical parameters are done with (25), (26) and (27). Besides, the parametrisation of the model depends on the variance of the transmitted samples, assumed to be unitary in the rest of the paper (i.e $\sigma_{\mathrm{Tx}}^{2}=1$ that leads to $P_{u}=2$ with (19) under the assumption of a normalized $\mathrm{Rx}$ filter $\left.h_{\mathrm{Rx}}(t)\right)$. Finally, as TxL is detrimental for the cell edges, where the power of the input signal is low [9] and thus the interference level is strong, we consider an initial SIR of $0 \mathrm{~dB}$ with $\sigma_{x_{b}}^{2}=10^{-8}$. 


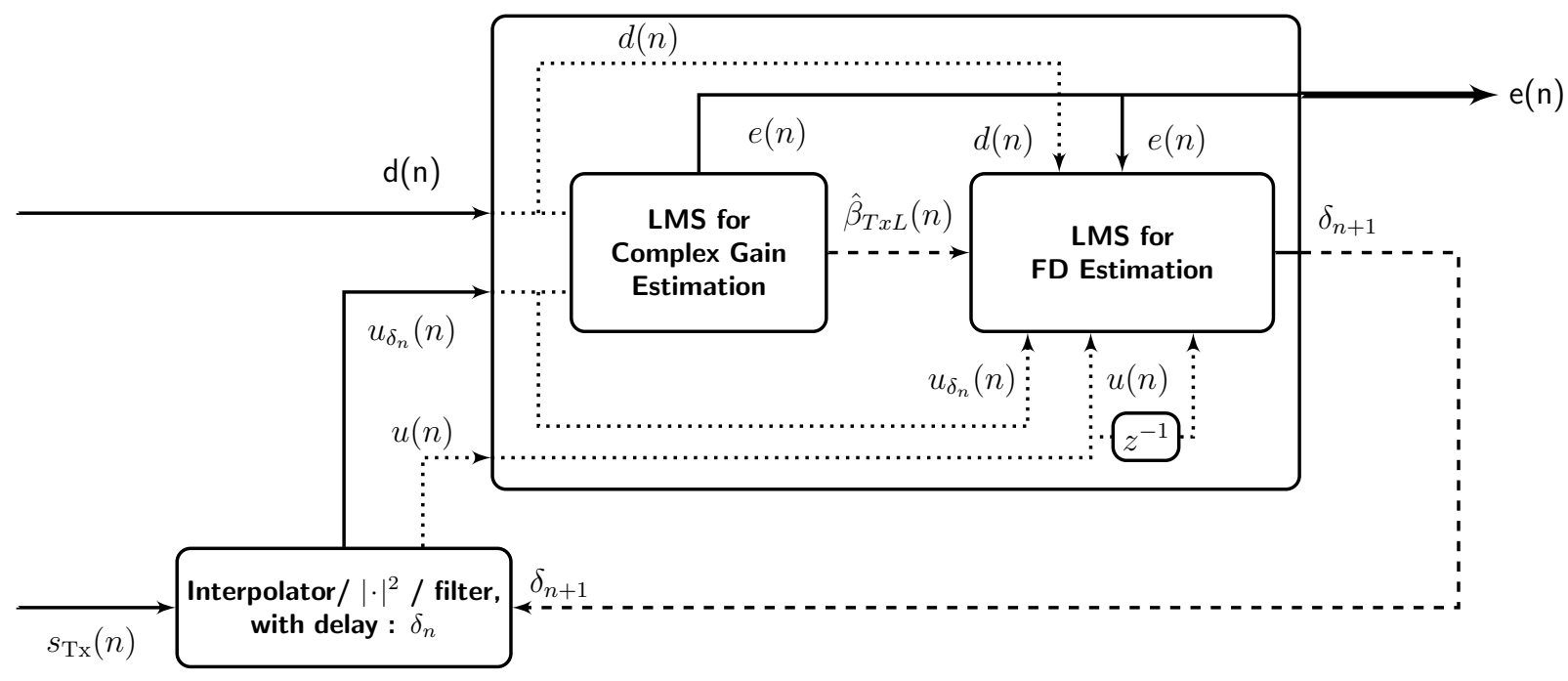

Figure 3: Scheme of the proposed joint estimation algorithm.

\subsection{Practical vs theoretical performance}

We first consider the synchronized case, in Figure 4, where the TxL channel is assumed to follow an AR1 model added to a constant value, as described in (12) and (13), and where the fractional delay is assumed to be perfectly known. The $K$ factor defined in (15) is set to 10 . We compare the asymptotic theoretical SIR to a simulated one versus the step size of the gain estimation algorithm, for several values of normalized Doppler frequency $f_{\mathrm{d}} T_{\mathrm{Rx}}$, and with the optimal step value described in (37). The range of normalized Doppler frequency is set to have a coherence time from approximatively $1 \mathrm{~ms}$ (related to worst case scenario in [18]) to several hundred seconds. It is shown that the simulated performance is coherent with the theory, and that the channel influence limits the asymptotic performance.

Figure 5 shows the evolution of the simulated maximal SIR versus the normalized Doppler frequency of the AR1 channel, with the Ricean factor $K=10$, and it validates the theory elaborated in (38). As seen in (25), the faster the channel, the lower the performance. In practice, assuming the knowledge of the statistics of the channel (AR1 variance $\sigma_{\alpha_{\mathrm{TxL}}}^{2}$, and state noise variance $\sigma_{\xi_{\mathrm{T} \times \mathrm{L}}}^{2}$ through $f_{\mathrm{d}} T_{\mathrm{Rx}}$ with $\left.(25)\right)$, the gain estimation process can be optimally tuned to provide the best asymptotic performance.

Figure 6 shows the performance of the LMS algorithm versus the step size of the algorithm for several values of $\mathrm{K}$ (which represents the ratio between the static and dynamic parts of the TxL channel, expressed in dB). We still consider an AR1 model to approximate the time-varying TxL channel, with $f_{\mathrm{d}} T_{\mathrm{Rx}}$ of $10^{-8}$. It is shown that the simulation agrees with the theory expressed in (33), and that in the case of a purely static channel $(K \rightarrow \infty)$, the performance of the LMS algorithm becomes linear with respect to the step size, as in this case, $Q\left(P_{u}, \sigma_{\alpha_{\mathrm{Tx}}}^{2}, \gamma\right)$

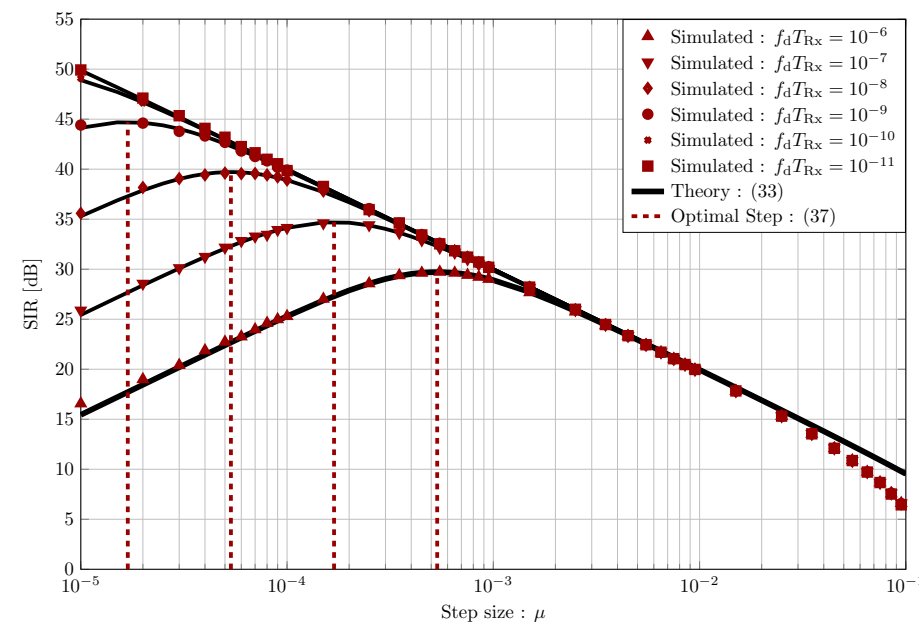

Figure 4: Comparison of the theoretical SIR obtained in (33) with the simulated asymptotic performance of the gain estimator, versus the step size for a TxL channel following an AR1 model of normalized Doppler frequency $f_{\mathrm{d}} T_{\mathrm{Rx}}$, in the synchronized case. The optimal step value of (37) is also computed.

is negligible. If the static part becomes negligible whith respect to the dynamic part (when $K$, expressed in $d B$, is negative), the performance reaches a boundary due to the impact of the factor $Q\left(P_{u}, \sigma_{\alpha_{\mathrm{T} \times \mathrm{L}}}^{2}, \gamma\right)$.

We now consider the impact of noncompensation of the fractional delay and the asymptotic performance of the joint estimation scheme. We still use an AR1 model for the TxL channel, as in (13), and $f_{\mathrm{d}} T_{\mathrm{Rx}}=10^{-9}$. Figure 7 shows the asymptotic performance of the LMS versus the step size, for several values of fractional delay, and we compare this to the synchronized asymptotic performance (33). Again, agreement with the theory described in (41) is shown, and it can be noted that in practice, a fractional delay will severely degrade the performance of the algorithm if it exceeds 0.02 , which means 2 percent of the $\mathrm{Rx}$ 


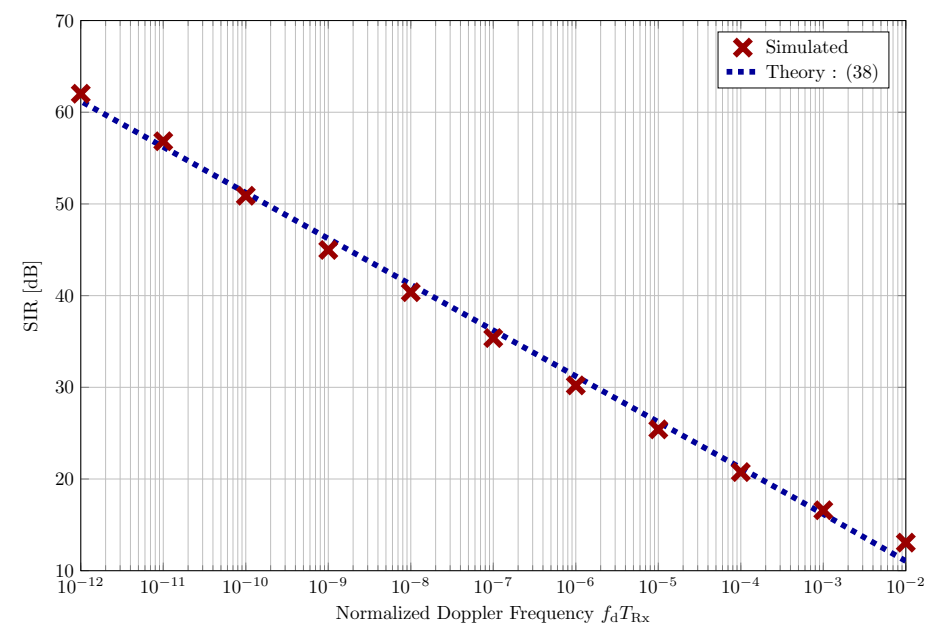

Figure 5: Comparison of the theoretical maximal SIR obtained in (38) with the simulated asymptotic performance of the gain estimator, versus the normalized Doppler frequency $f_{\mathrm{d}} T_{\mathrm{Rx}}$, in the synchronized case.

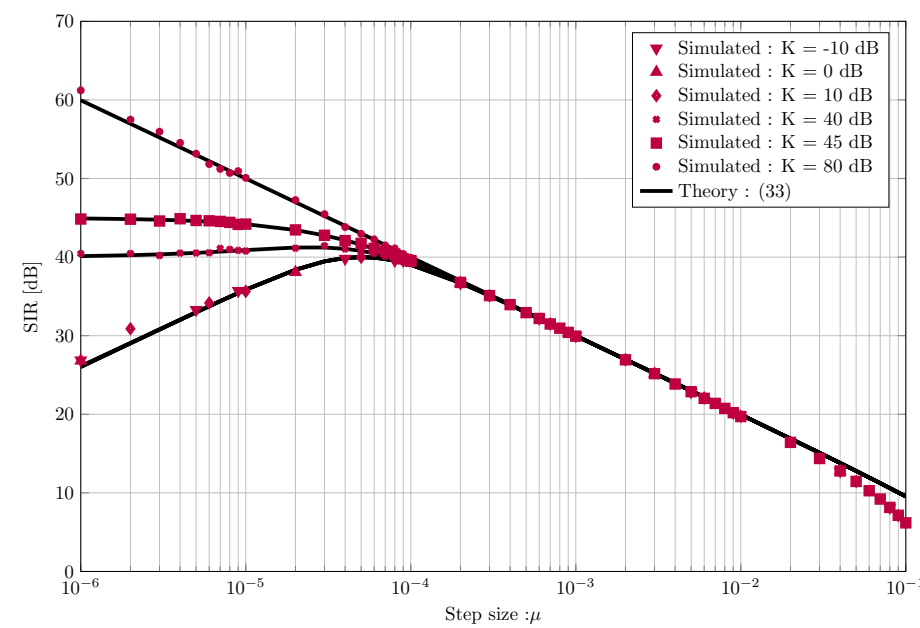

Figure 6: Comparison of the theoretical SIR obtained in (33) with the simulated asymptotic performance of the gain estimator, versus the step size for a TxL channel with $\mathrm{SIR}=0 \mathrm{~dB}$ and different values of $K$.

sampling time.

This justifies that a joint estimation scheme must be investigated if the fractional delay is unknown. Figure 7 shows the asymptotic performance of the joint estimation algorithm versus the step size of the gain estimator process, for $\nu=10^{-6} / \sigma_{x_{b}}^{2}$. It is shown that this algorithm provides a very good asymptotic performance for different values of fractional delay, as it almost reaches the boundary defined by the theoretical synchronized performance (33). It is also shown that the performance of the proposed algorithm is independent from the FD value, as the performance with the joint estimation algorithm is similar in the range of the simulated FDs.

From Figure 4 to Figure 6, the desired signal was a complex Gaussian white signal. We recall that the assumption of an uncorrelated (stationary) signal has been

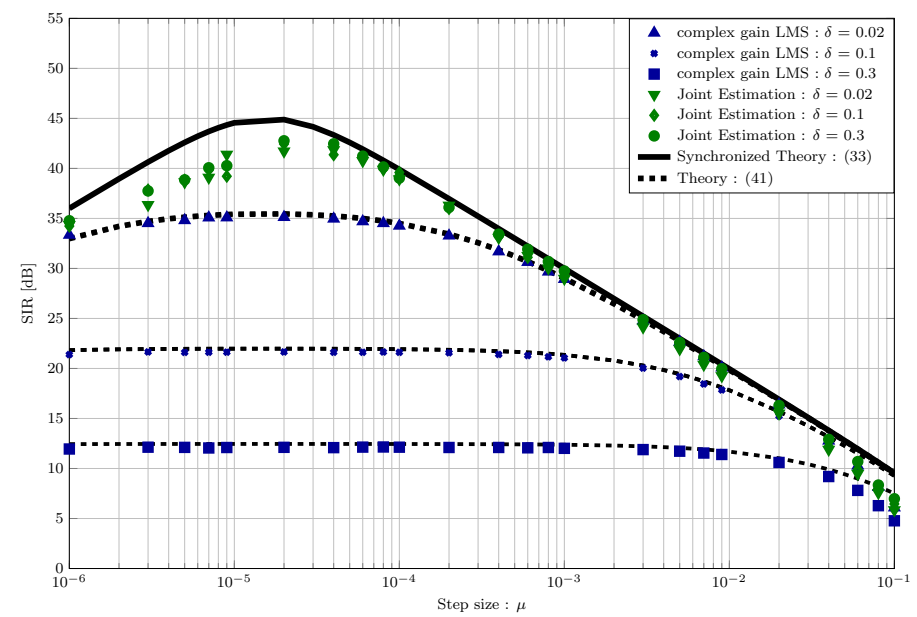

Figure 7: Comparison of the theoretical SIR obtained in (41) with the simulated performance of the gain estimator without compensation of the fractional delay, and with the joint estimation algorithm, versus the step size of the gain estimator algorithm for different values of fractional delay $\delta$.

made to derive the analytic performance formulae, and we propose in Figure 8 to validate also the theoretical performance on a typical (cyclostationary) signal used in communication systems. We use an orthogonal multiplexing division multiplexing (OFDM) signal with 72 quadrature phase-shift keying (QPSK) subcarriers, a fast Fourier transform of size of 128, and a cyclic prefix of size nine samples for both transmission and reception. We display the asymptotic simulated performances for different fractional delays versus the step-size of the joint estimation algorithm. It is shown that, for this particular use-case, the simulated results corroborate with the theory that was obtained for a white signal in (41).

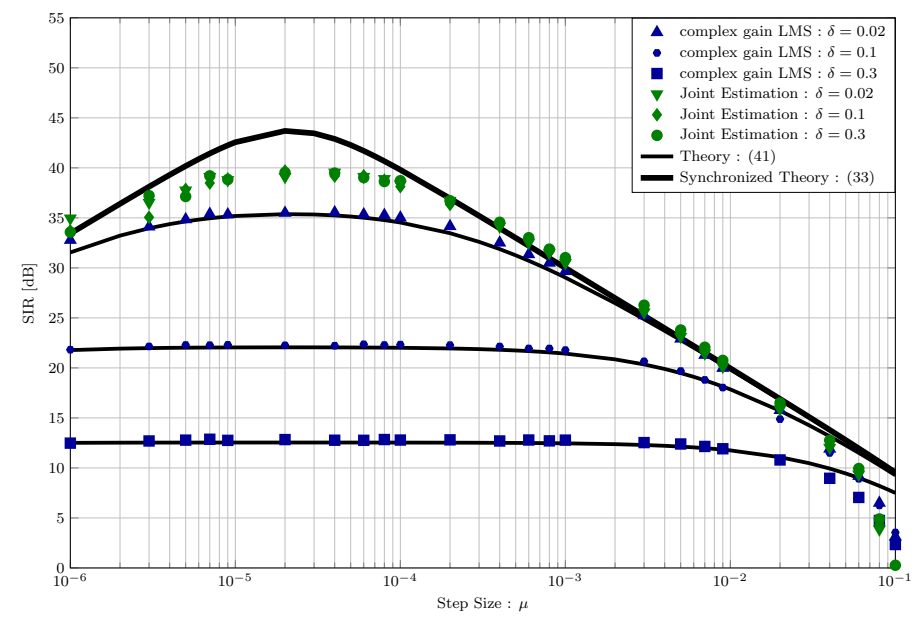

Figure 8: Comparison of the theoretical SIR obtained in (41) with the simulated performance of the gain estimator without compensation of the fractional delay, and with the joint estimation algorithm, versus the step size of the gain estimator algorithm for different values of fractional delay $\delta$ for an OFDM signal. 


\subsection{Bit error rate simulation}

Up to now, we have discussed the performance in terms of the SIR for both correlated and uncorrelated signals. We now look at bit error rate simulations. For this, we assume a FDD framework where the Tx and Rx signals share the same modulation parameters, and where the TxL channel is assumed to be a complex AR1 channel with a fractional delay between the Rx path and the Tx path of 0.2 samples. We synthesize a reference $u(n)$ following $(19)$ with power of $2\left(\operatorname{as} \sigma_{\mathrm{Tx}}^{2}=1\right)$, and we assume a power entry signal of $-80 \mathrm{~dB}$ (cell edge context) with $K=10$ and $f_{\mathrm{d}} T_{\mathrm{Rx}}=10^{-9}$.

\subsubsection{The wideband code division multiple access (WCDMA) context}

We first consider a WCDMA framework based on quadrature phase-shift keying modulation. The code is an orthogonal variable spreading factor code of length 16 , and a scrambling sequence of size 38400 is also used. Square root raised cosine filters are used both in transmission and reception, with a roll-off factor of 0.22 . We show in Figure 9 the results of the bit error rate performance without the compensation system and with a joint estimation compensation scheme (with $\mu=2 \cdot 10^{-5}$ and $\eta=10^{-6}$ ) for different values of $E b / N_{o}$ (where $E b$ is the energy per bit, and $N_{0}$ is the white additive noise spectral density), and for different SIRs. Here, the initial SIRs are computed from only the power of the desired signal, without taking into account the additive white noise, to have constant $\mathrm{TxL}$ pollution power versus the channel signal-to-noise ratio. We show that our method greatly reduces the bit error rate, and that the compensated bit error rate comes close to the ideal channel influence $Q\left(\sqrt{2 E_{\mathrm{b}} / N_{0}}\right)$.

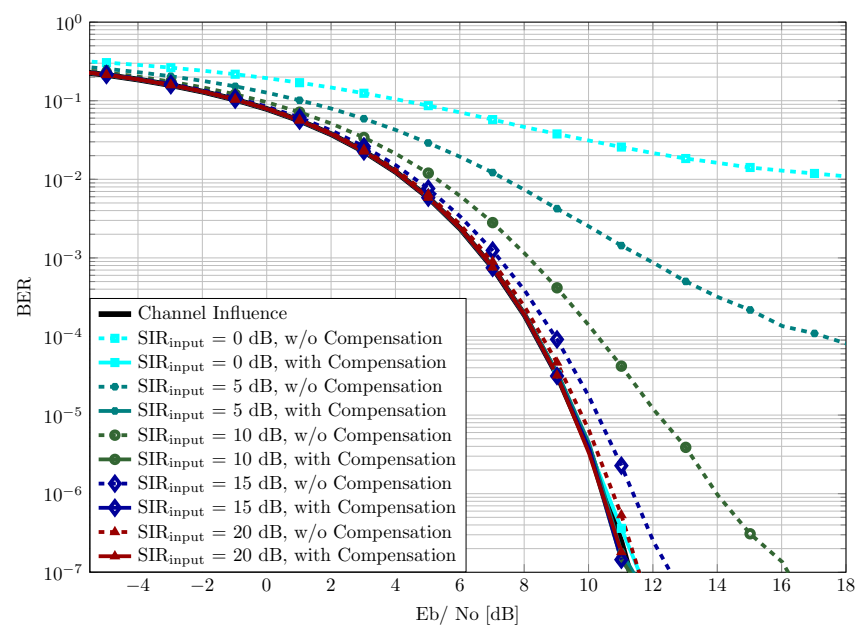

Figure 9: Bit error rate without and with compensation for a wideband code division multiple access scheme, for different $E b / N_{0}$.

\subsubsection{The OFDM context}

We now compare the bit error rate for the case of simplified long-term evolution (LTE) modulation that is based on the third generation partnership project model [28]. The data signal follows LTE 1.4 MHz bandwidth specifications based on OFDM, with 72 quadrature phaseshift keying subcarriers, a fast Fourier transform of size 128 , and a cyclic prefix of length of nine samples. The bit error-rate is computed on the uncoded bits of the data and through an additive white Gaussian noise channel with various signal-to-noise ratios. We consider that the noisy signal is polluted by $\mathrm{TxL}$ and that the transmitted signal has the same modulation parameters. Figure 10 shows the results of the bit error rate without compensation and with the joint estimation scheme (with $\mu=2 \cdot 10^{-5}$ and $\eta=10^{-6}$ ) for different values of the channel signal-to-noise ratio and for different SIRs. The theoretical performance without $\mathrm{TxL}$ (i.e., only the influence of the channel) is shown in black in Figure 10. Again, it shows that TxL has a detrimental impact on performance, and that the proposed system greatly reduces the bit error rate.

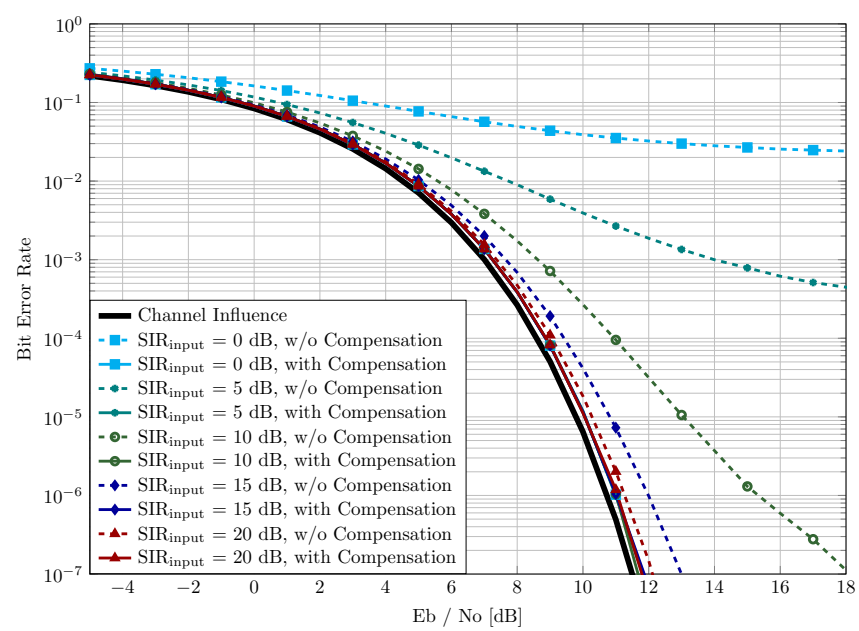

Figure 10: Bit error rate without and with compensation for OFDM modulation, for different $E b / N_{0}$.

\subsection{Discussion of the channel model}

In this paper, we have assumed a frequency flat channel for the duplexer that is equivalent to consider a Dirac distribution as a time impulse response. As a consequence, the compensation scheme aims to estimate the amplitude of this Dirac and its fractional delay. Frequency flat duplexer channels are often used in the literature, as for example by $[16,5,29,22]$, and this hypothesis can be partially justified through a typical isolation profile, as described for example by [30], which shows only a little attenuation variation in the transmission and reception bands (typically less than $2 \mathrm{~dB}$ ). However, a purely frequency flat channel is a limiting case, and as in practice, it is equivalent to an estimation of only the mean contribution of the filter, with the other contributions of the TxL filter being neglected. A more realistic assumption would be to consider a quasi-flat TxL channel i.e., a filter with a main but nonunique component in its time impulse response. 
From the initial profile, we propose to synthesize a filter that respects the initial pattern proposed by [30]. We next apply our method on a polluting model, where the complex gain is replaced by the synthesized filter. We assume here nontime-varying filter coefficients and an important oversampling factor. The frequency and time impulse responses obtained are shown in the Figure 11.

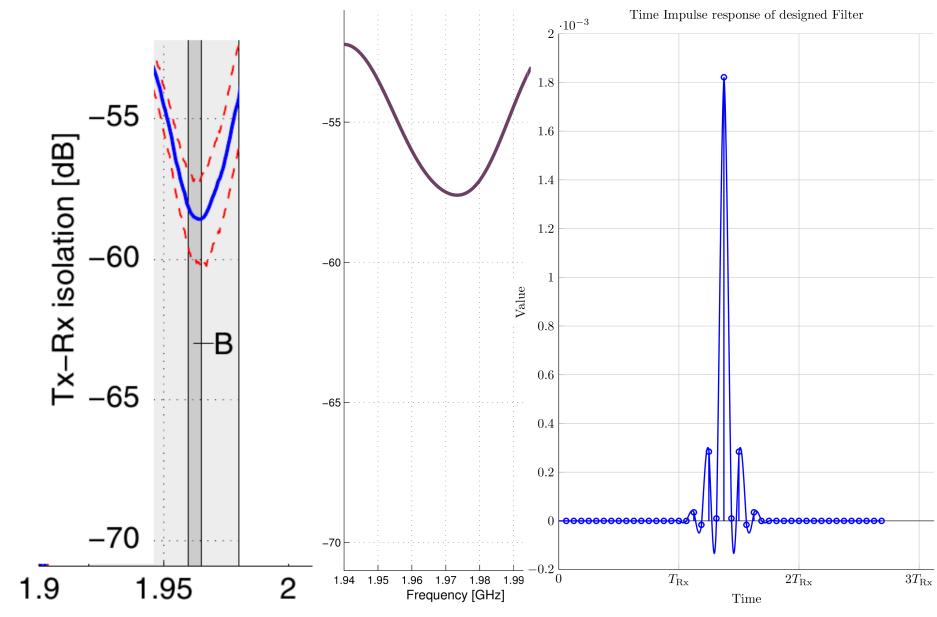

Figure 11: Left: Typical isolation profile from [30]. Middle: Frequency profile of the designed filter. Right: Time impulse response of the designed filter.

The synthesized time impulse response is narrow with respect to the receiver sampling time $T_{\mathrm{Rx}}$, which is coherent with a quasi-flat channel assumption. The proposed joint estimation algorithm estimates and suppresses the most important component of the filter impulse response, and we show in Figure 12 the convergence of the complex gain and the fractional delay, and the mean performance of the structure. This shows that even in a quasi-flat channel, the proposed structure has good performance and can reduce the influence of the TxL pollution. In such a case, the fractional delay estimated by our algorithm is expected to converge to the position of the maximum of the time impulse response of the synthesized filter (this position is denoted as 'expected fractional delay'). We want to now compare our method with the TxL compensation method presented by [15] (and variations of this method can be found in $[16,22,31]$ ), based on a multitap LMS canceller. Figure 13 compares the performances of this multitapLMS (with 8 taps) and the performance of the proposed scheme for the synthesized filter, with several values of the expected fractional delay. It can be seen that the proposed scheme has better asymptotic performance than the multitap LMS, especially in the case of a nonnegligible fractional delay value. We finally compare in table 3 the complexity (real operators) of the proposed JE algorithm, in terms of additions, multiplications and shifts (as the steps $\mu$ and $\eta$ are tuned to a power of 2) and a comparison with the classical multi-tap LMS versus the number of the taps and applied for 8 taps, as used in Figure 13. It is shown that the proposed method, in addition to have better asymp-
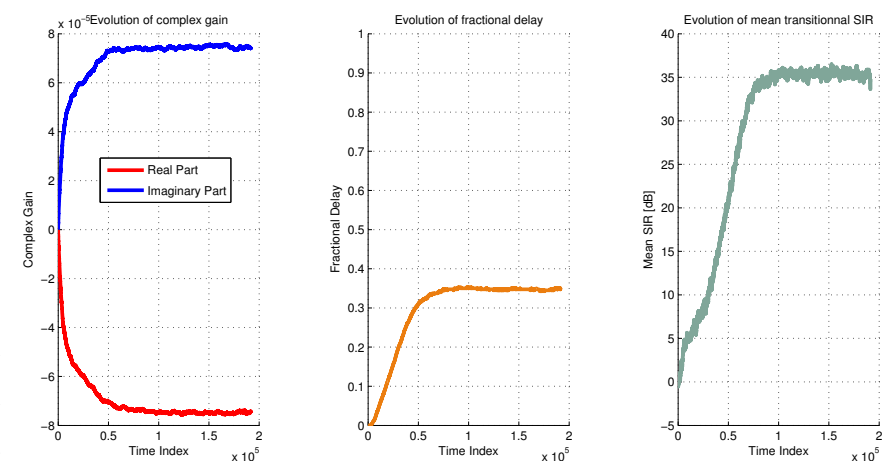

Figure 12: Left: Evolution of the convergence mode of the complex gain estimation process. Middle: Evolution of the convergence mode of the fractional delay estimation process. Right: Evolution of the mean performance of the cancellation structure.

totic performance than classical multi-tap approach even in the proposed frequency selective TxL channel scenario, also offers a lower computational complexity.

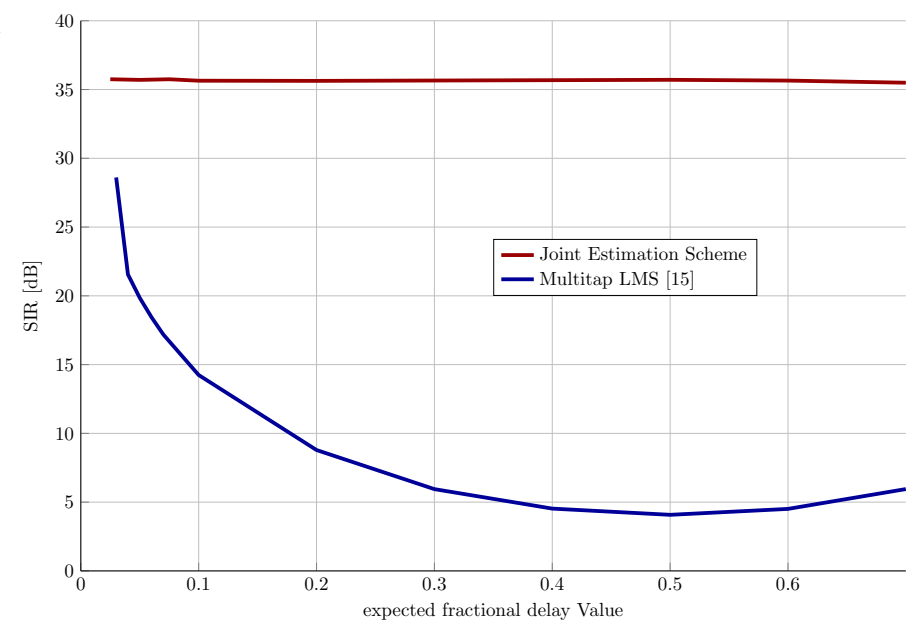

Figure 13: Comparison between the asymptotic performance of a classic multi-tap LMS scheme ([15]) and the proposed scheme, when the TxL filter is selective with different values of the expected fractional delay.

\section{Conclusion}

This study focuses on joint estimation of fractional delay and channel gain for compensation for TxL pollution in FDD transceivers. We consider a time-varying TxL channel that is assumed to follow a first-order auto-regressive model added to a constant value, and we consider the presence of a fractional delay to model propagation effects of the analog and digital parts. We first derived analytic formulae of the asymptotic signal-to-interference ratio of a gain estimator based on a LMS approach in the synchronized case, and we have shown that the time-varying channel limits the asymptotic performance. Then, we expressed the influence of the fractional delay in terms of 


\begin{tabular}{|c|c|c||c|c|}
\hline \multirow{2}{*}{} & \multicolumn{2}{|c||}{ Proposed Algorithm } & \multicolumn{2}{c|}{ Multi-tap LMS with $P$ taps } \\
\cline { 2 - 5 } & $\mathbf{L}$ & $\mathbf{L}=\mathbf{8}$ & $\mathbf{P}$ & $\mathbf{P}=\mathbf{8}$ \\
\hline+ & $11+\mathrm{L}$ & 19 & $4 \mathrm{P}+2$ & 38 \\
\hline $\mathrm{x}$ & $20+\mathrm{L}$ & 28 & $4 \mathrm{P}$ & 36 \\
\hline Shifts & 6 & 6 & $2 \mathrm{P}$ & 18 \\
\hline
\end{tabular}

Table 3: Complexity comparison between the proposed algorithm and classical multi-tap LMS. $L$ is the size of the fractional delay interpolation filter

asymptotic SIR, to show that it must be estimated upstream in the compensation stage. We finally proposed a compensation algorithm based on joint estimation of the fractional delay and the complex channel gain. This adaptive algorithm is recursive and online, and it proposes good asymptotic performance with low complexity. We also tested the behavior of the proposed method in the case of a selective TxL channel case, and we have shown that the method is robust and provides better performance than a classical multi-tap LMS approach. As a perspective to this work, the model of the Tx-leakage channel could be completed to take into account the delay spread of this channel, in addition to the coherence time. This could be useful when the TxL channel exhibits a strong frequency selectivity.

\section{A. Proof of (33)}

We introduce the difference between the ideal coefficient $\beta_{\mathrm{TxL}}(n)$ and the iterative coefficient $\hat{\beta}_{\mathrm{TxL}}(n)$, called the misalignment error $v(n)$ [32]:

$$
v(n)=\hat{\beta}_{\mathrm{TxL}}(n)-\beta_{\mathrm{TxL}}(n) .
$$

From (45) using (20), (17), (29) and (31), $\epsilon(n)$ can be expressed as

$$
\epsilon(n)=-v(n) u_{\delta}(n)
$$

and the SIR can be expressed as a function of the mean square misalignment:

$$
\operatorname{SIR}_{\text {comp }}=-10 \log _{10}\left[\frac{P_{u} P_{v}}{\sigma_{x_{b}}^{2}}\right]
$$

where $P_{u}$ is the power of the reference defined in (19), and $P_{v}$ is the mean square misalignment. With (12), (13), (17)-(32), the recursive expression of the misalignment is:

$$
\begin{aligned}
v(n+1)= & \left(1-\mu\left|u_{\delta}(n)\right|^{2}\right) v(n)+\mu u_{\delta}(n) x_{b}(n) \\
& -\xi_{\operatorname{TxL}}(n+1)+(1-\gamma) \alpha_{\operatorname{TxL}}(n) .
\end{aligned}
$$

As $\xi_{\mathrm{TxL}}(n)$ and $x_{b}(n)$ are assumed to be white uncorrelated processes, the mean square misalignment can be expressed as:

$$
\begin{aligned}
P_{v}= & \left(1-\mu P_{u}\right)^{2} P_{v}+\mu^{2} P_{u} \sigma_{x_{b}}^{2}+2(1-\gamma) \sigma_{\alpha_{\mathrm{T} \times \mathrm{L}}}^{2} \\
& +2\left(1-\mu P_{u}\right)(1-\gamma) E\left[\left|\alpha_{\mathrm{T} \times \mathrm{L}}(n) v(n)\right|\right] .
\end{aligned}
$$

Using both recursive expressions of $\alpha_{\mathrm{TxL}}(n)$ and $v(n)$, defined in (13) and (48), respectively, we have:

$$
E\left[\left|\alpha_{\mathrm{TxL}}(n) v(n)\right|\right]=\frac{-(1-\gamma) \sigma_{\alpha_{\mathrm{TXL}}}^{2}}{1-\gamma\left(1-\mu P_{u}\right)},
$$

and replacing (50) in (49), we have

$$
\begin{aligned}
P_{v}\left[\mu P_{u}\left(2-\mu P_{u}\right)\right]= & \mu^{2} P_{u} \sigma_{x_{b}}^{2}+2(1-\gamma) \times\left[\sigma_{\alpha_{\mathrm{T} \times \mathrm{L}}}^{2}\right. \\
& \left.-\frac{\left(1-\mu P_{u}\right)(1-\gamma) \sigma_{\alpha_{\mathrm{T} \times \mathrm{L}}}^{2}}{1-\gamma\left(1-\mu P_{u}\right)}\right]
\end{aligned}
$$

which is equivalent to

$$
\begin{aligned}
P_{v}= & \frac{\mu \sigma_{x_{b}}^{2}}{2-\mu P_{u}}+\frac{2 \sigma_{\alpha_{\mathrm{T \times L}}}^{2}(1-\gamma)}{\mu P_{u}\left(2-\mu P_{u}\right)\left(1-\gamma\left(1-\mu P_{u}\right)\right)} \times \\
& {\left[1-\gamma\left(1-\mu P_{u}\right)-\left(1-\mu P_{u}\right)(1-\gamma)\right], }
\end{aligned}
$$

and the final expression of the mean square misalignment is finally

$$
P_{v}=\frac{\mu \sigma_{x_{b}}^{2}}{2-\mu P_{u}}+\frac{2 \sigma_{\alpha_{\mathrm{T} \times \mathrm{L}}}^{2}(1-\gamma) \mu P_{u}}{\mu P_{u}\left(2-\mu P_{u}\right)\left(1-\gamma\left(1-\mu P_{u}\right)\right)} .
$$

Finally, by reintroducing (51) in (47), we obtain the asymptotic SIR defined in (33) and the channel variations impact factor $Q\left(P_{u}, \sigma_{\alpha_{\mathrm{T} \times \mathrm{L}}}^{2}, \gamma\right)$ described in (34).

\section{B. Approximation of the fractional delay noise vari- ance}

If we assume linear interpolation for $u_{\delta}(n)$, we have:

$$
u_{\delta_{n}}(n)=(1-\delta) u(n)+\delta u(n-1)
$$

and from (39), we also have:

$$
\eta_{\mathrm{TxL}}(n)=u_{\delta_{n}}(n)-u(n)=-\delta[u(n)-u(n-1)]
$$

From the reference generation of (19), as the reference is a chi-squared distributed, we have $E[|u(n)|]=\sigma_{\text {Tx }}^{2}$ and $P_{u}=2 \sigma_{\mathrm{Tx}}^{4}$, with $\sigma_{\mathrm{Tx}}^{2}$ as the variance of the transmitted samples. Thus:

$$
\begin{aligned}
\sigma_{\eta_{\mathrm{T} \times \mathrm{L}}}^{2}= & E\left[\left|\eta_{\mathrm{T} \times \mathrm{L}}(n)\right|^{2}\right] \\
= & \delta^{2} E\left[|u(n)-u(n-1)|^{2}\right] \\
= & \delta^{2}\left(E\left[|u(n)|^{2}\right]+E\left[|u(n-1)|^{2}\right]-\right. \\
& 2 E[|u(n) u(n-1)|])
\end{aligned}
$$


Assuming indendence between $u(n)$ and $u(n-1)$,

$$
\begin{aligned}
\sigma_{\eta_{\mathrm{T} \times \mathrm{L}}}^{2}= & \delta^{2}(\underbrace{E\left[|u(n)|^{2}\right]}_{2 \sigma_{\mathrm{TX}}^{4}}+\underbrace{E\left[|u(n-1)|^{2}\right]}_{2 \sigma_{\mathrm{T \times}}^{4}}- \\
& 2 \underbrace{E[|u(n)|]}_{\sigma_{\mathrm{T}}^{2}} \underbrace{E[|u(n-1)|]}_{\sigma_{\mathrm{Tx}}^{2}}) \\
= & 2 \delta^{2} \sigma_{\mathrm{Tx}}^{4}=\delta^{2} P_{u}
\end{aligned}
$$

\section{Proof of (41)}

In the case of a noncompensated fractional delay, from (20) and (45), the estimation error becomes:

$$
\epsilon(n)=-v(n) u(n)+\beta_{\mathrm{TxL}}(n) \eta_{\mathrm{TxL}}(n),
$$

and the SIR can be expressed as:

$$
\operatorname{SIR}_{\text {comp }}=-10 \log _{10}\left[\frac{P_{u} P_{v}}{\sigma_{x_{b}}^{2}}+\left(\left|\beta_{0_{\mathrm{T} \times \mathrm{L}}}\right|^{2}+\sigma_{\alpha_{\mathrm{T} \times \mathrm{L}}}^{2}\right) \frac{\sigma_{\eta_{\mathrm{T} \times \mathrm{L}}}^{2}}{\sigma_{x_{b}}^{2}}\right] .
$$

With the same demonstration process that led to (48), the recursive expression of the misalignment can be expressed as:

$$
\begin{aligned}
v(n+1)= & \left(1-\mu|u(n)|^{2}\right) v(n)+\mu u(n) x_{b}(n) \\
& -\xi_{\mathrm{TxL}}(n)+(1-\gamma) \alpha_{\mathrm{TxL}}(n) \\
& +\mu u(n) \beta_{\mathrm{TxL}}(n) \eta_{\mathrm{TxL}}(n+1) .
\end{aligned}
$$

Assuming that $\eta_{\mathrm{TxL}}(n)$ is white additive zero-mean Gaussian noise, of variance $\sigma_{\eta_{\mathrm{T \times L}}}^{2}$, and with (34), the power of the misalignment can be expressed as:

$$
P_{v}=\begin{gathered}
\left(1-\mu P_{u}\right)^{2} P_{v}+\mu^{2} P_{u} \sigma_{x_{b}}^{2} \\
+\mu^{2} P_{u} \sigma_{\eta_{\mathrm{T} \times \mathrm{L}}}^{2} \sigma_{\beta_{\mathrm{T} \times \mathrm{L}}}^{2}+Q\left(P_{u}, \sigma_{\alpha_{\mathrm{T} \times \mathrm{L}}}^{2}, \gamma\right),
\end{gathered}
$$

and reintroducing (57) in (55) leads to the asymptotic SIR performance described in (41).

\section{LMS approach for fractional delay estimation}

We use a stochastic gradient descent to update the fractional delay estimation, with the same cost function $J(n)$ as in the gain estimator:

$$
\begin{aligned}
& J(n)=E\left[|e(n)|^{2}\right] \\
& \delta_{n+1}=\delta_{n}-\nu \frac{\partial J(n)}{\partial \delta}
\end{aligned}
$$

with $\nu$ the constant step size of the fractional delay estimation process. By minimizing the instantaneous power of the output $e(n)$ which is a complex signal [33], the update becomes:

$$
\begin{aligned}
\frac{\partial J(n)}{\partial \delta} & =2 \Re\left(e^{*}(n) \frac{e(n)}{\partial \delta}\right) \\
& =-\hat{\beta}_{T x L}(n) \frac{\partial u_{\delta_{n}}(n)}{\partial \delta}-u_{\delta_{n}}(n) \frac{\partial \hat{\beta}_{T x L}(n)}{\partial \delta} .
\end{aligned}
$$

We consider the approximation:

$$
\frac{\partial u_{\delta_{n}}(n)}{\partial \delta} \approx \Delta_{u}(n)=u(n-1)-u(n),
$$

which is the first-order Taylor decomposition of the derivative component $\partial u_{\delta_{n}}(n) / \partial \delta$. We denote $L(n)=\partial \hat{\beta}_{T x L}(n) / \partial \delta$, and the recursive expression of $L(n)$ can be obtain with (32) as:

$$
\begin{gathered}
L(n+1)=\left(1-\mu\left|u_{\delta_{n}}(n)\right|^{2}\right) L(n)+2 \mu u_{\delta_{n}}(n) \Delta_{u}(n) \\
-\mu d(n) \Delta_{u}(n) .
\end{gathered}
$$

From (59) and (60), the update of the fractional delay estimation is:

$$
\delta_{n+1}=\delta_{n}+\nu \Re\left\{\left[\hat{\beta}_{T x L}(n) \Delta_{u}(n)+u_{\delta_{n}}(n) L(n)\right] e^{*}(n)\right\} .
$$

And finally, the fractional delay estimation algorithm is set from (61)-(63).

\section{References}

[1] R. Gerzaguet, L. Ros, F. Belveze, J.-M. Brossier, Joint estimation of complex gain and fractional delay for Tx leakage compensation in FDD transceivers., in: Proc. 21st International Conference on Electronics Circuits and Systems (ICECS), 2014.

[2] M. Meurer, P. Baier, T. Weber, Y. Lu, A. Papathanassiou, Joint transmission: advantageous downlink concept for CDMA mobile radio systems using time division duplexing, Electronics Letters vol 36 (2000) pp 900-901.

[3] H. Martikainen, Analysis of duplexing modes in the IEEE 802.16 wireless system, in: Proc. European Wireless Conference (EW), 2010, pp. 849-856.

[4] I. Masafumi, J. Tsutsumi, Y. Endo, H. Nakamura, Y. Satoh, An attenuation improvement technology for ladder SAW/FBAR filters and duplexers employing cancellation circuit, in: Proc. 41st European Microwave Conference (EuMC), 2011, pp. 751754.

[5] A. Frotzscher, G. Fettweis, A stochastic gradient LMS algorithm for digital compensation of Tx leakage in Zero-IF-Receivers, in: Proc. IEEE Vehicular Technology Conference (VTC), 2008, pp. 1067-1071.

[6] B. Razavi, Design considerations for direct-conversion receivers, IEEE Transactions on Circuits and Systems (Analog and Digital Signal Processing) vol 44 (1997) pp 428-435.

[7] D. Manstretta, M. Brandolini, F. Svelto, Second-order intermodulation mechanisms in CMOS downconverters, IEEE Journal of Solid-State Circuits vol 38 (2003) pp 394-406.

[8] C. Lederer, M. Huemer, The influence of DC offsets on the digital cancellation of second-order Tx intermodulation distortions in homodyne receivers, in: Proc. IEEE International Conference on Wireless Information Technology and Systems (ICWITS), 2012, pp. 1-4.

[9] A. Kiayani, L. Anttila, M. Valkama, Modeling and dynamic cancellation of Tx-Rx leakage in FDD transceivers, in: Proc IEEE 56th International Midwest Symposium on Circuits and Systems (MWSCAS), 2013, pp. 1089-1094.

[10] M. Brandolini, P. Rossi, D. Manstretta, F. Svelto, Toward multistandard mobile terminals - fully integrated receivers requirements and architectures, IEEE Transactions on Microwave Theory and Techniques 53 (2005) 1026-1038.

[11] K. Dufrene, R. Weigel, Highly linear IQ downconverter for reconfigurable wireless receivers, in: The European Conference on Wireless Technology, 2005, pp. 19-22.

[12] S.-S. Lee, J. Lee, I.-Y. Lee, S.-G. Lee, J. Ko, A new Tx leakagesuppression technique for an RFID receiver using a dead-zone amplifier, in: Proc. IEEE International Solid-State Circuits Conference (ISSCC), 2013, pp. 92-93. 
[13] G. Fettweis, M. Lohning, D. Petrovic, M. Windisch, P. Zillmann, W. Rave, Dirty RF: a new paradigm, in: Proc. IEEE 16th International Symposium on Personal, Indoor and Mobile Radio Communications (PIMRC), 2005, pp. 2347-2355 Vol. 4.

[14] V. Aparin, A new method of Tx leakage cancelation in WCDMA and GPS receivers, in: Proc IEEE Radio Frequency Integrated Circuits Symposium (RFIC), 2008, pp. 87-90.

[15] M. Kahrizi, J. Komaili, J. Vasa, D. Agahi, Adaptive filtering using LMS for digital Tx IM2 cancellation in WCDMA receiver, in: Proc. IEEE Radio and Wireless Symposium, 2008, pp. 519522 .

[16] M. Faulkner, DC offset and IM2 removal in direct conversion receivers, IEE Proceedings on Communications vol 149 (3) (2002) pp 179-184.

[17] A. Frotzscher, G. Fettweis, Least squares estimation for the digital compensation of $\mathrm{Tx}$ leakage in zero-IF receivers, in: Proc. IEEE Global Telecommunications Conference (GLOBECOM), 2009, pp. 1-6.

[18] A. Frotzscher, D. Plettemeier, G. Fettweis, On the time variance of Tx leakage in FDD Zero-IF Transceivers, in: Proc. IEEE 70th Vehicular Technology Conference Fall (VTC 2009-Fall), 2009, pp. 1-5.

[19] R. Gerzaguet, L. Ros, F. Belveze, J.-M. Brossier, On the performance of digital adaptive spur cancellation for multi-standard radio frequency transceivers, Digital Signal Processing 33 (2014) Pages 83-97.

[20] U. Mengali, A. D'Andrea, Synchronization Techniques for Digital Receivers, Applications of Communications Theory, Springer, 1997.

[21] T.-K. Nguyen, C.-H. Kim, G.-J. Ihm, M.-S. Yang, S.-G. Lee, CMOS low-noise amplifier design optimization techniques, IEEE Transactions on Microwave Theory and Techniques 52 (5) (2004) 1433-1442.

[22] C. Lederer, M. Huemer, LMS based digital cancellation of second-order $\mathrm{Tx}$ intermodulation products in homodyne receivers, in: Proc. IEEE Radio and Wireless Symposium (RWS), 2011, pp. 207-210.

[23] Y. Grenier, Time-dependent ARMA modeling of nonstationary signals, IEEE Transactions on Acoustics, Speech and Signal Processing vol 31 (1983) pp 899-911.

[24] S. Ghandour-Haidar, L. Ros, J.-M. Brossier, On the use of firstorder autoregressive modeling for Rayleigh flat fading channel estimation with Kalman filter, Signal Processing vol 92 (2012) pp 601-606.

[25] T. Laakso, V. Valimaki, M. Karjalainen, U. Laine, Splitting the unit delay [FIR/all pass filters design], IEEE Signal Processing Magazine vol 13 (1) (1996) pp 30-60.

[26] V. Valimaki, A. Haghparast, Fractional delay filter design based on truncated Lagrange interpolation, Signal Processing Letters, IEEE vol 14 (2007) pp 816-819.

[27] B. Widrow, J. McCool, M. Ball, The complex LMS algorithm, Proceedings of the IEEE 63 (4) (1975) $719-720$.

[28] 3rd Generation Partnership Project, Evolved universal terrestrial radio access (e-utra) ; physical channels and modulation, Tech. rep., 3GPP TS (2011).

[29] A. Frotzscher, G. Fettweis, Tx leakage impact on analog-digital conversion in zero-IF receivers, in: Proc. IEEE 70th Vehicular Technology Conference Fall (VTC 2009-Fall), 2009, pp. 1-5.

[30] A. Frotzscher, G. Fettweis, Baseband analysis of Tx leakage in WCDMA zero-IF-receivers, in: 3rd International Symposium on Communications, Control and Signal Processing (ISCCSP), 2008, pp. 129-134.

[31] A. Frotzscher, G. Fettweis, Digital compensation of transmitter leakage in FDD zero-IF receivers, Transactions on Emerging Telecommunications Technologies vol 23 (2012) pp 105-120.

[32] S. Haykin, Adaptive filter theory, Prentice Hall, 1996.

[33] D. Brandwood, A complex gradient operator and its application in adaptive array theory, IEE Proceedings $\mathrm{H}$ (Microwaves, Optics and Antennas) vol 130 (1) (1983) pp 11-16. 